\title{
Die österreichische private Krankenversicherung - Ein Monopol?
}

\author{
Christine Duller \\ Johannes Kepler Universität Linz
}

\begin{abstract}
The Austrian Private Health Insurance - a Monopoly? This paper considers the concentration in the Austrian private health-insurance market between 1992 and 2001. The calculation is based on premiums earned and takes into account groups of companies and shows that in deed there is a monopoly since 1996. A discussion about reasons and effects is added.
\end{abstract}

Zusammenfassung: Anhand einiger Konzentrationsmaßzahlen und eines Dominanzmaßes wird die Konzentration am österreichischen privaten Krankenversicherungsmarkt in den Jahren 1992 bis 2001 beurteilt. Die Untersuchung erfolgt auf Basis der abgegrenzten Gesamtprämien unter Berücksichtigung von Konzernzugehörigkeiten und zeigt, dass der Markt seit 1996 eine monopolartige Struktur aufweist. Eine Diskussion der Ursachen und Folgen dieser Marktstruktur rundet den Beitrag ab.

Keywords: Concentration, Private Health-Insurance Market, Monopoly.

\section{Einführung}

Im Mittelpunkt dieser Arbeit steht der österreichische private Krankenversicherungsmarkt, insbesondere die zentrale Frage, ob dieser Markt monopolartige Strukturen aufweist. Die Marktsituation der privaten Versicherungen ist im Zentrum vieler Analysen, eine mögliche und häufig verwendete Kennzahl zur Beschreibung der Marktsituation ist der Anteil der Prämien am BIP. Dieser banal klingende Ansatz birgt schon die ersten Probleme in sich, die in einer eigenen Studie zu diesem Thema aufgearbeitet wurden (Url, 1996).

In Anlehnung an diese gesamtversicherungswirtschaftlichen Betrachtungen zeigt Tabelle 1 den jeweiligen Anteil der Krankenversicherungsprämien am BIP in den Jahren 1992 bis 2001. Als Vergleichsgröße wurde das BIP herangezogen, genauer formuliert das nominelle Bruttoinlandsprodukt zu laufenden Preisen berechnet nach dem Konzept des Europäischen Systems Volkswirtschaftlicher Gesamtrechnungen (Quelle: eigene Berechnung und Darstellung mit Daten aus: Bundesministerium für Finanzen 1993 Tabelle 10A/1, Bundesministerium für Finanzen 1994 Tabelle 10/1, Bundesministerium für Finanzen 1995 Tabelle 10/1, Bundesministerium für Finanzen 1996 Tabelle 10/1, Bundesministerium für Finanzen 1997 Tabelle 10/1, Bundesministerium für Finanzen 1998 Tabelle 10/1, Statistik Austria 2003 Tabelle 15.01 S. 285, Tabelle 10 für die Jahre 1998 bis 2001, http://www.fma.gv.at/de/fma/statisti/versiche/oesterre.htm, 7.10.2005).

Als Prämien werden die abgegrenzten Gesamtprämien verwendet (Angaben in Euro wurden bei Bedarf in ATS umgerechnet), diese enthalten sowohl die direkten als auch die indirekten Prämien. Die abgegrenzten Prämien bilden die Basis der betriebswirtschaftlichen Betrachtung der Unternehmensergebnisse eines Versicherungsunternehmens, 
Tabelle 1: Gesamtprämie der Krankenversicherung und BIP

\begin{tabular}{cccc}
\hline Jahr & $\begin{array}{c}\text { Abgegrenzte Gesamtprämie } \\
\text { in Tausend ATS }\end{array}$ & $\begin{array}{c}\text { BIP } \\
\text { in Mio. ATS }\end{array}$ & $\begin{array}{c}\text { Prämie } \\
\text { in \% des BIP }\end{array}$ \\
\hline 1992 & 13.924 .713 & 2.089 .240 & $0.67 \%$ \\
1993 & 14.984 .696 & 2.159 .528 & $0.69 \%$ \\
1994 & 15.505 .496 & 2.276 .105 & $0.68 \%$ \\
1995 & 15.973 .503 & 2.370 .721 & $0.67 \%$ \\
1996 & 15.692 .984 & 2.449 .953 & $0.64 \%$ \\
1997 & 15.357 .936 & 2.511 .062 & $0.61 \%$ \\
1998 & 15.340 .425 & 2.623 .098 & $0.58 \%$ \\
1999 & 15.584 .964 & 2.711 .660 & $0.57 \%$ \\
2000 & 16.019 .599 & 2.843 .855 & $0.56 \%$ \\
2001 & 16.683 .937 & 2.924 .215 & $0.57 \%$ \\
\hline
\end{tabular}

weil sie als periodengerecht erfasster Betrag der Ausgangspunkt für Analysen der versicherungstechnischen und nichtversicherungstechnischen Rechnung sind (Bundesministerium für Finanzen, 1998). Die Vorteile und Nachteile der Verwendung von direkten Prämien anstelle von Gesamtprämien werden in der Studie von Thomas Url (1996) genauer erläutert, für die Analyse der Marktkonzentration im Bereich der privaten Krankenversicherung ist es allerdings unerheblich, ob direkte oder Gesamtprämien verwendet werden, weil die indirekte Prämie einen verschwindenden Anteil an der Gesamtprämie einnimmt, wie aus Tabelle 2 ersichtlich ist.

Tabelle 2: Gesamtprämie und direkte Prämie in der Krankenversicherung

\begin{tabular}{cccc}
\hline Jahr & $\begin{array}{c}\text { Abgegrenzte Gesamtprämie } \\
\text { in Tausend ATS }\end{array}$ & $\begin{array}{c}\text { Davon indirekt } \\
\text { in Tausend ATS }\end{array}$ & $\begin{array}{c}\text { Anteil } \\
\text { indirekt }\end{array}$ \\
\hline 1992 & 13.924 .713 & 11.539 & $0.08 \%$ \\
1993 & 14.984 .696 & 13.065 & $0.09 \%$ \\
1994 & 15.505 .496 & 6.522 & $0.04 \%$ \\
1995 & 15.973 .503 & 5.348 & $0.03 \%$ \\
1996 & 15.692 .984 & 11.820 & $0.08 \%$ \\
1997 & 15.357 .936 & 11.263 & $0.07 \%$ \\
1998 & 15.340 .425 & 15.820 & $0.10 \%$ \\
1999 & 15.584 .964 & 14.536 & $0.09 \%$ \\
2000 & 16.019 .599 & 18.165 & $0.11 \%$ \\
2001 & 16.683 .937 & 19.980 & $0.12 \%$ \\
\hline
\end{tabular}

(Quelle: eigene Berechnung und Darstellung mit Daten aus: Bundesministerium für Finanzen 1993 Tabelle 10A/1, Bundesministerium für Finanzen 1994 Tabelle 10/1, Bundesministerium für Finanzen 1995 Tabelle 10/1, Bundesministerium für Finanzen 1996 Tabelle 10/1, Bundesministerium für Finanzen 1997 Tabelle 10/1, Bundesministerium für Finanzen 1998 Tabelle 10/1, http://www.fma.gv.at/de/fma/statisti/versiche/oesterre.htm 
21.2.2005, Tabelle 10 für die Jahre 1998 bis 2001)

Der Anteil der Gesamtprämie am BIP wird auch als Versicherungsdurchdringung bezeichnet, daher zeigt Tabelle 1, dass die Versicherungsdurchdringung bezüglich der Krankenversicherung im beobachteten Zeitraum tendenziell gesunken ist.

\section{Der Begriff Monopol}

Ein Gütermarkt wird als Monopolmarkt bezeichnet, wenn er die folgenden drei Eigenschaften aufweist (Herberg, 1989):

1. Am Markt treten viele kleine Nachfrager, aber nur ein Anbieter auf.

2. Für das betreffende Gut gibt es keine oder nur schlechte Alternativen.

3. Der Preis des Gutes wird ausschließlich vom Markt bestimmt.

Der Begriff Monopol ist demnach sehr strikt und daher für Krankenversicherungen nicht passend, da einerseits mehrere Anbieter am Markt auftreten, andererseits der Preis nicht ausschließlich vom Markt bestimmt wird, weil die Tariffestlegung von Versicherungen gesetzlichen Bestimmungen unterliegt.

Etwas schwächer ist die Begriffsbestimmung von marktbeherrschenden Unternehmen, wie sie das Kartellgesetz vorsieht: Hier wird ein Unternehmen dann als marktbeherrschend eingestuft, wenn einerseits kein oder nur wenig Wettbewerb existiert und andererseits das Unternehmen im Verhältnis zu anderen Mitbewerbern eine überragende Marktstellung hat. Eine Abgrenzung von marktbeherrschenden Unternehmern kann über die Marktanteile getroffen werden, beispielsweise wird ein Unternehmen ab einem Marktanteil von 30\% als marktbeherrschend eingestuft (Kartellgesetz, 1988).

In dieser Untersuchung soll daher der verwendete Begriff monopolartige Struktur zum Ausdruck bringen, dass ein Unternehmen den Markt beherrscht, aber trotzdem kein Monopol im strengen Sinn vorliegt.

\section{Konzentrationsmessung}

Die statistische Konzentrationsmessung gibt Auskunft über die Art der Zuordnung eines Merkmales auf seine Merkmalsträger, wobei das Merkmal in Form von relativen Anteilen an der Merkmalssumme erfasst wird. Sind die Merkmalsträger ebenfalls relativ erfasst, spricht man von relativer Konzentration oder Disparität, ansonsten von absoluter Konzentration. Für die Messung von Unternehmenskonzentration ist in erster Linie die absolute Konzentration von Interesse, weil hier auch die absolute Anzahl der Unternehmen am Markt berücksichtigt wird (Häni, 1987).

Dies ist unmittelbar aus der folgenden Überlegung einsichtig: Angenommen das Marktvolumen verteilt sich immer gleich auf die gesamte Zahl der Anbieter, dann ist die Konzentration in einem Markt mit drei Anbietern sicher höher zu bewerten als in einem Markt mit 3.000 Anbietern. Als geometrische Grundlage der Konzentrationsmessung dient die Konzentrationskurve (Marfels, 1977). 
In dieser Untersuchung wird nicht nur die statische Konzentration von Interesse sein, sondern auch die Veränderung der Konzentration im Zeitverlauf. Daher werden die verschiedenen Konzentrationsmaßzahlen für die Jahre 1992 bis 2001 errechnet und auf eine zeitliche Veränderung hin untersucht. Neben verschiedenen Konzentrationsmaßzahlen wird auch ein Dominanzmaß berechnet, welches zusätzliche Information über die Struktur des Marktes vermitteln soll. Im Falle eines stark konzentrierten Marktes wird damit die Frage beantwortet, ob der Markt eher monopolartige oder oligopolartige Strukturen aufweist (Häni, 1987).

\subsection{Merkmalsträger und Merkmal}

Die Merkmalsträger in dieser Untersuchung sind Unternehmen, die eine wirtschaftliche Einheit darstellen. Um ein objektives Kriterium verwenden zu können, wird ein Unternehmen einem anderen als wirtschaftliche Einheit zugeordnet, wenn das Beteiligungsverhältnis am Kapital mehr als 50\% ausmacht. Versicherungsunternehmen in Österreich können ausschließlich in Form einer Aktiengesellschaft oder in Form eines Vereines auf Gegenseitigkeit geführt werden (Versicherungsaufsichtsgesetz, 1978).

Die meisten Versicherungsunternehmen sind in Form einer Aktiengesellschaft geführt, damit lassen sich die Beteiligungsverhältnisse aus den Geschäftsberichten der jeweiligen Unternehmen entnehmen. Beteiligungen unter 50\% werden in dieser Untersuchung vernachlässigt, obwohl außer Frage steht, dass es auch hier zu wirtschaftlichen Abhängigkeiten kommen kann, die möglicherweise den Markt beeinflussen.

Das Kartellgesetz legt fest, dass bei Versicherungsunternehmen die Prämieneinnahmen als Grundlage von Berechnungen im Rahmen des Kartellgesetztes heranzuziehen sind. Als Merkmal werden daher die abgegrenzten Gesamtprämien der Krankenversicherung herangezogen (Kartellgesetz, 1988).

\subsection{Verwendete Konzenrationsmaße}

Im Folgenden sei

$n \quad$ die Anzahl der Merkmalsträger

$p_{i} \quad$ der Anteil des Merkmalsträgers i an der gesamten Merkmalssumme mit $0<p_{i} \leq 1$ für $i=1, \ldots, n$ und $\sum_{i=1}^{n} p_{i}=1$

$p_{j} \quad$ der Anteil des Merkmalsträgers $\mathrm{j}$ an der gesamten Merkmalssumme bei nach dem Anteil absteigend geordneten Merkmalsträgern

$j \quad$ der Rang der nach absteigendem Anteil geordneten Merkmalsträger

$m$ die Anzahl der berücksichtigten größten Merkmalsträger, $m \leq n$

\section{Konzentrationsrate}

Die Konzentrationsrate (= Konzentrationskoeffizient) errechnet sich gemäß

$$
C R m=\sum_{j=1}^{m} p_{j} .
$$


Die Vorteile der Konzentrationsrate liegen in der leichten Verständlichkeit, der leichten Verfügbarkeit und dem direkten Bezug zur Konzentrationskurve. Beispielsweise bedeutet $C R 3=0.5$, dass die drei größten Merkmalsträger 50\% der Merkmalssumme auf sich vereinen. Leichte Verfügbarkeit ist gewährleistet, weil man nur die Angaben der m größten Merkmalsträger sowie die gesamte Merkmalssumme zur Berechnung benötigt. Die Konzentrationsrate bringt aber auch verschiedene Nachteile mit sich. Sie weist nur einen beschränkten Informationsgehalt auf, wobei sich dieser Nachteil mindern lässt, indem man für verschiedene $m$ die Konzentrationsraten bestimmt. Weitere Nachteile liegen in der willkürlichen Wahl von $\mathrm{m}$ und darin, dass Merkmalsverschiebungen oberhalb der $\mathrm{m}$ größten Merkmalsträger unberücksichtigt bleiben. Auch kleinere Transfers zwischen den $m$ größten Merkmalsträgern, die keine Verschiebungen in der Rangreihenfolge bewirken, verändern die Konzentrationsrate nicht. Darüber hinaus werden kleine und große Merkmalsträger innerhalb der m größten Merkmalsträger gleich gewichtet (Häni, 1987 und Marfels, 1977).

\section{Herfindahl-Index}

Der Herfindahl-Index (= Hirschman-Herfindahl-Index) errechnet sich gemäß

$$
H E R=\sum_{i=1}^{n} p_{i}^{2} .
$$

Die Vorteile liegen in der einfachen Konstruktion und in der anschaulichen Interpretation als gewichtete durchschnittliche Steigung der Konzentrationskurve, wobei die Gewichtung über die einzelnen Anteile der Merkmalsträger erfolgt (Adelman, 1969). Da jeder einzelne Merkmalsträger berücksichtigt wird, können auch kleine Veränderungen wahrgenommen werden, die beispielsweise von der Konzentrationsrate nicht erkannt werden. Kleine und große Merkmalsträger werden unterschiedlich gewichtet, und zwar nach ihren relativen Marktanteilen. In der unterschiedlichen Gewichtung liegt aber auch ein Nachteil, weil kleine und große Merkmalsträger sehr stark unterschiedlich gewichtet werden und daher der Index von sehr vielen sehr kleinen Merkmalsträgern nahezu unbeeinflusst bleibt (Häni, 1987). Der Wertebereich erstreckt sich theoretisch auf das Intervall [0, 1], in der Praxis sind aber die ermittelten Werte auch bei hoher Konzentration relativ gering, es kann zu einer Unterschätzung der Konzentration kommen (Horvath, 1970 und Marfels, 1977).

\section{Erweiterter Herfindahl-Index}

Der erweiterte Herfindahl-Index (= verallgemeinerter Herfindahl-Index)

$$
H E R_{\alpha}=\sqrt[\alpha-1]{\sum p_{i}^{\alpha}}=\left(\sum p_{i}^{\alpha}\right)^{1 /(a-1)}
$$

führt für $\alpha=2$ auf den üblichen Herfindahl-Index, für $\alpha \rightarrow \infty$ fällt der erweiterte Herfindahl-Index mit der Konzentrationsrate für den größten Merkmalsträger $C R 1 \mathrm{zu}$ sammen (Hannah and Kay, 1977). Für $\alpha=1$ erhält man den Exponential-Index. Wählt man einen Wert $\alpha>2$, führt dies zu einer höheren Gewichtung größerer Merkmalsträger, für $\alpha<2$ erhält man dem entsprechend eine höhere Gewichtung der kleinen Merkmalsträger. 
Als Vorteil ist anzusehen, dass (je nach Fragestellung) eine individuelle Gewichtung über Wahl von $\alpha$ möglich ist. Darin liegt aber auch gleichzeitig der Nachteil, weil somit die Wahl von $\alpha$ willkürlich erfolgt (Deffaa, 1982, Häni, 1987 und Pisch and Schmidt, 1983).

\section{Entropie-Maß}

Für das Entropie-Maß

$$
E N T=-\sum_{i=1}^{n} p_{i} \ln \left(p_{i}\right)
$$

kann die Basis des Logarithmus beliebig gewählt werden. Das Entropie-Maß ist ein inverses Konzentrationsmaß: Bei vollständiger Konzentration (= Monopol) ist die Entropie gleich Null, bei vollständiger Gleichverteilung des Marktes auf $n$ Anbieter nimmt die Entropie ihr von der Anzahl der Markteilnehmer abhängiges Maximum $\ln (n)$ an. Je mehr Anbieter sich den Markt teilen, desto höher ist die maximale Entropie und desto geringer die Marktkonzentration. Im Vergleich zum Herfindahl-Index werden kleinere Merkmalsträger stärker berücksichtigt. Ein weiterer Vorteil liegt in der guten Desaggregationseigenschaft, denn bei Aufteilung der Merkmalsträger in Gruppen lässt sich die Entropie errechnen als Summe der Entropien zwischen den Gruppen und der gewichteten Entropien innerhalb der Gruppen. Dadurch können Auswirkungen von Fusionierungen quantitativ erfasst werden. Ein Nachteil liegt in der schlechten Vergleichbarkeit mit anderen Konzentrationsmaßen, die aus der Tatsache resultiert, dass im Gegensatz zu den anderen Konzentrationsmaßen das Entropie-Maß eine inverse Maßzahl ist. Darüber hinaus ist die maximale Entropie abhängig von der Anzahl der Anbieter, daher sind auch Entropiemaße untereinander am besten über die relative Entropie (= Quotient aus Entropie und maximaler Entropie) vergleichbar (Häni, 1987, Hart, 1971, Marfels, 1971, Spätling, 1970 und Theil, 1967).

\section{Exponential-Index}

Der Exponential-Index

$$
E X P=\prod_{i=1}^{n} p_{i}^{p_{i}}
$$

weist folgenden Zusammenhang mit dem Entropie-Maß auf

$$
E N T=-\sum_{i=1}^{n} p_{i} \ln \left(p_{i}\right)=-\ln \left(\prod_{i=1}^{n} p_{i}^{p_{i}}\right)=-\ln (E X P)
$$

Der Exponential-Index weist die Vorteile des Entropie-Maßes auf, bietet aber noch zusätzliche Vorteile durch Normierung und direkte Konzentrationsmessung statt inverser, die guten Desaggregationseigenschaften bleiben erhalten. Auch hier besteht die Tendenz zur Unterschätzung der Konzentration (Bruckmann, 1969, Häni, 1987, Marfels, 1971 und Marfels, 1977).

\section{Rosenbluth-Index}

Der Rosenbluth-Index (= Hall-Tidemann-Index) errechnet sich gemäß 


$$
R O S=\frac{1}{2 \sum_{j=1}^{n} j p_{j}-1} .
$$

Der Rosenbluth-Index berücksichtigt die Anzahl der Merkmalsträger und ihren Rang, kleinere Merkmalsträger erhalten somit mehr Gewicht. Ähnlich wie bei der Berechnung der Konzentrationsrate müssen auch hier die Merkmalsträger vor der Berechnung nach absteigendem Marktanteil geordnet werden. Es besteht ein direkter Bezug zur Konzentrationskurve, denn

$$
\sum_{j=1}^{n} j p_{j}-\frac{1}{2}
$$

entspricht der Fläche oberhalb der Konzentrationskurve. Auch der Rosenbluth-Index führt zur Unterschätzung der Konzentration (Hall and Tidemann, 1967, Häni, 1987, Piesch, 1975 und Rosenbluth, 1961).

\subsection{Verwendetes Dominanzsmaß}

Als Dominanzmaß wird das Herfindahl-Dominanz-Maß (= Deeffaa-Maß) verwendet, für dessen Berechnung die Gruppe der $m$ größten Unternehmen bzw. die Gruppe der (n-m) kleinsten Unternehmen jeweils als homogen betrachtet wird. Dies hat zur Folge, dass der durchschnittliche Marktanteil eines großen Unternehmens $C R m / m$ beträgt, analog dazu ist der Marktanteil eines kleinen Unternehmens mit $(1-C R m) /(n-m)$ anzusetzen. Das Dominanzmaß errechnet sich mit diesen Annahmen analog zum Herfindahl-Index (5) und lässt sich in vereinfachter Form anschreiben als

$$
D E F_{m}=\frac{(C R m)^{2}}{m}+\frac{(1-C R m)^{2}}{n-m}
$$

Jenes $m *$, für das $D E F_{m}$ sein Maximum annimmt, trennt die Oligopolgruppe von der Verfolgergruppe, damit bilden die $m *$ größten Unternehmen ein Oligolpol (Deffaa, 1982 und Eurich et al., 1997).

\section{Private Krankenversicherung in Österreich}

In den Jahren 1992 bis 1998 gab es neun Unternehmen und in den Jahren 1999 bis 2001 acht Unternehmen in der Krankenversicherung (Bundesministerium für Finanzen, 2001).

In einem ersten Schritt wurden die Strukturen der einzelnen Unternehmen untersucht um Unternehmenseinheiten herausfiltern zu können. Ausgangspunkt waren die in der Statistik 1992 angeführten Krankenversicherer, deren Namen in weiterer Folge nur noch in Kurzformen angeführt werden:

1. Austria-Collegialität Österreichische Versicherung Aktiengesellschaft

2. Wiener Städtische Allgemeine Versicherung Aktiengesellschaft

3. Versicherungsanstalt der österreichischen Bundesländer Versicherungsaktiengesellschaft 
4. Merkur Versicherung Aktiengesellschaft

5. Erste Allgemeine Versicherungs-Aktiengesellschaft

6. Interunfall Versicherung Aktiengesellschaft

7. Wiener Allianz Versicherungs-Aktiengesellschaft

8. Volksfürsorge-Jupiter Allgemeine Versicherungs-Aktiengesellschaft (kurz: VJV)

9. Collegialität Versicherung Aktiengesellschaft

Bei der Untersuchung wurden folgende Verflechtungen berücksichtigt:

- Erste Allgemeine und Generali treten ab 1987 gemeinsam als EA-Generali auf.

- Collegialität ist seit 1991 eine Tochter der Austria-Collegialität.

- Interunfall gehört seit 1991 zum Generali-Konzern.

- BARC entsteht 1996 aus Bundesländer und Austria-Collegialität.

- Aus BARC wird 1999 UNIQA.

- VJV wird 2001 Wüstenrot.

In Tabelle 3 und Tabelle 4 sind die Marktanteile an den abgegrenzten Gesamtprämien in der österreichischen Krankenversicherung für die Jahre 1992 bis 2001 unter Berücksichtigung von Konzernzugehörigkeiten angegeben (Quelle: eigene Berechnung und Darstellung mit Daten aus: Bundesministerium für Finanzen 1993 Tabelle 10A/1, Bundesministerium für Finanzen 1994 Tabelle 10/1, Bundesministerium für Finanzen 1995 Tabelle 10/1, Bundesministerium für Finanzen 1996 Tabelle 10/1, Bundesministerium für Finanzen 1997 Tabelle 10/1, Bundesministerium für Finanzen 1998 Tabelle 10/1, http://www.fma.gv.at/de/fma/statisti/versiche/oesterre.htm 21.2.2005, Tabelle 10 für die Jahre 1998 bis 2001).

Tabelle 3: Marktanteile 1992-1996

\begin{tabular}{clrrrrr}
\hline \multirow{2}{*}{ Nr. } & \multirow{2}{*}{ Unternehmenseinheit } & \multicolumn{5}{c}{ Marktanteile Krankenversicherung } \\
& & 1992 & 1993 & 1994 & 1995 & 1996 \\
\hline 1 & Austria-Collegialität/BARC & $32.5 \%$ & $32.1 \%$ & $31.9 \%$ & $31.5 \%$ & $51.1 \%$ \\
2 & Wiener Städtische & $20.9 \%$ & $20.6 \%$ & $20.5 \%$ & $20.5 \%$ & $20.6 \%$ \\
3 & Bundesländer & $19.6 \%$ & $19.6 \%$ & $19.8 \%$ & $19.8 \%$ & \\
4 & Merkur & $13.3 \%$ & $13.7 \%$ & $13.6 \%$ & $13.6 \%$ & $13.6 \%$ \\
5 & Generali & $11.2 \%$ & $11.6 \%$ & $11.9 \%$ & $12.1 \%$ & $12.2 \%$ \\
6 & Allianz & $2.0 \%$ & $1.9 \%$ & $1.9 \%$ & $1.9 \%$ & $1.9 \%$ \\
7 & VJV & $0.5 \%$ & $0.5 \%$ & $0.5 \%$ & $0.5 \%$ & $0.5 \%$ \\
\hline \multicolumn{2}{c}{ Summe } & $100.0 \%$ & $100.0 \%$ & $100.0 \%$ & $100.0 \%$ & $100.0 \%$ \\
\hline
\end{tabular}


Tabelle 4: Marktanteile 1997-2001

\begin{tabular}{|c|c|c|c|c|c|c|}
\hline \multirow{2}{*}{ Nr. } & \multirow{2}{*}{ Unternehmenseinheit } & \multicolumn{5}{|c|}{ Marktanteile Krankenversicherung } \\
\hline & & 1997 & 1998 & 1999 & 2000 & 2001 \\
\hline 1 & BARC/UNIQA & $50.8 \%$ & $50.4 \%$ & $50.0 \%$ & $49.7 \%$ & $49.5 \%$ \\
\hline 2 & Wiener Städtische & $20.7 \%$ & $20.9 \%$ & $20.9 \%$ & $21.0 \%$ & $20.9 \%$ \\
\hline 3 & Merkur & $13.6 \%$ & $13.7 \%$ & $13.8 \%$ & $13.9 \%$ & $13.9 \%$ \\
\hline 4 & Generali & $12.4 \%$ & $12.6 \%$ & $12.8 \%$ & $12.8 \%$ & $13.0 \%$ \\
\hline 5 & Allianz & $1.8 \%$ & $1.8 \%$ & $2.0 \%$ & $2.1 \%$ & $2.2 \%$ \\
\hline 6 & VJV & $0.6 \%$ & $0.5 \%$ & $0.5 \%$ & $0.5 \%$ & $0.5 \%$ \\
\hline & Summe & $100.0 \%$ & $100.0 \%$ & $100.0 \%$ & 100.0 & $100.0 \%$ \\
\hline
\end{tabular}

Die Veränderungen in den Jahren 1992 bis 1995 sind eher geringfügig. Die AustriaCollegialität ist mit einem ca. ein Drittel Marktanteil die führende Unternehmenseinheit. Wesentliche Marktanteile weisen auch Wiener Städtische und Bundesländer mit jeweils etwa $20 \%$ auf. Im Bereich von ca. 12\% sind Merkur und Generali, die beiden restlichen Unternehmenseinheiten Allianz und VJV weisen einen sehr geringen Marktanteil auf. Durch Zusammenschluß der Austria-Collegialität und der Bundesländer im Jahr 1996 zur BARC wird diese Unternehmenseinheit eindeutig Marktführer mit einem Marktanteil von über 50\%. Dieser Marktanteil sinkt zwar bis zum Jahr 2001 unter die 50\%-Marke, ist aber trotzdem noch deutlich größer als die einzelnen Marktanteile aller anderen Unternehmen.

In Abbildung 1 sind die Konzentrationskurven der Jahre 1992 und 2001 dargestellt, im Vergleich der beiden Kurven ist bereits die steigende Konzentration zu erkennen.

Der graue Bereich oberhalb der Konzentrationskurve entspricht dem halben Nenner des Rosenbluth-Index (8), eine Reduktion dieser Fläche deutet auf eine steigende Konzentration hin. Der schraffierte Bereich zwischen Konzentrationskurve und der $45^{\circ}$-Linie entspricht der Konzentrationsfläche, deren Zuwachs ebenfalls auf die steigende Konzentration hinweist.

Die errechneten Maßzahlen können aus Tabelle 5 und Tabelle 6 entnommen werden. Zur Berechnung wurden immer die in absteigender Reihenfolge geordneten Marktanteile verwendet, obwohl diese Anordnung nur für die Konzentrationsrate (1) und den Rosenbluth-Index (7) notwendig ist. Bei allen anderen Indizes ergibt sich durch diese Anordnung keine Änderung.

In den Jahren 1992 bis 1995 sind nur unwesentliche Veränderungen erkennbar, die Schwankungen in den Marktanteilen sind sehr gering und dem entsprechend gibt es auch bei den Konzentrationsmaßen kaum eine Veränderung. Das Deffaa-Maß kann eine Oligopolgruppe mit drei Unternehmen herauskristallisieren. Bemerkenswert ist die Veränderung im Jahr 1996. Durch den Zusammenschluss der Austria-Collegialität und Bundesländer zur BARC (dann UNIQA) kommt es im Jahr 1996 zu einer Monopolstellung am Krankenversicherungsmarkt, rund die Hälfte des gesamten Marktvolumens liegt in den Händen eines Konzerns.

Vergleicht man insbesondere die Veränderungen in den diversen Konzentrationsmaßzahlen zwischen den Jahren 1995 und 1996, so muss man allerdings feststellen, dass sich diese doch sehr einschneidende Marktveränderung in den meisten Maßzahlen nur sehr 


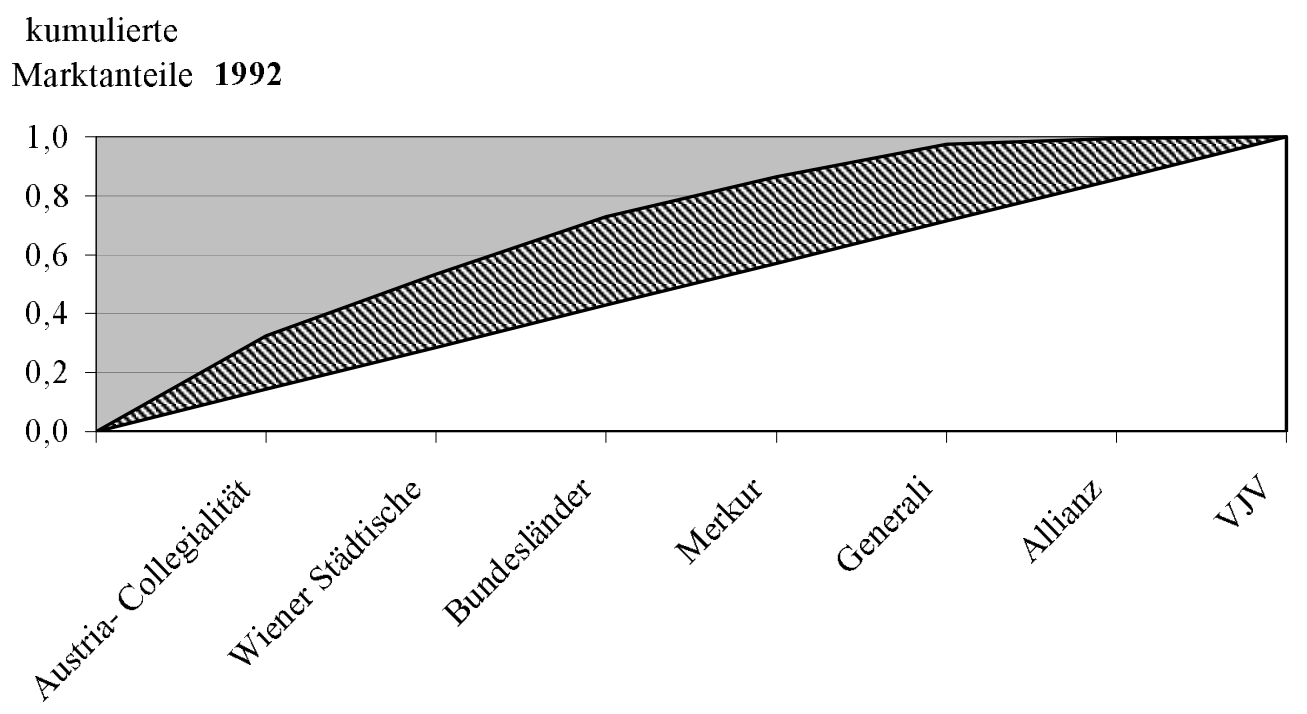

kumulierte

Marktanteile 2001

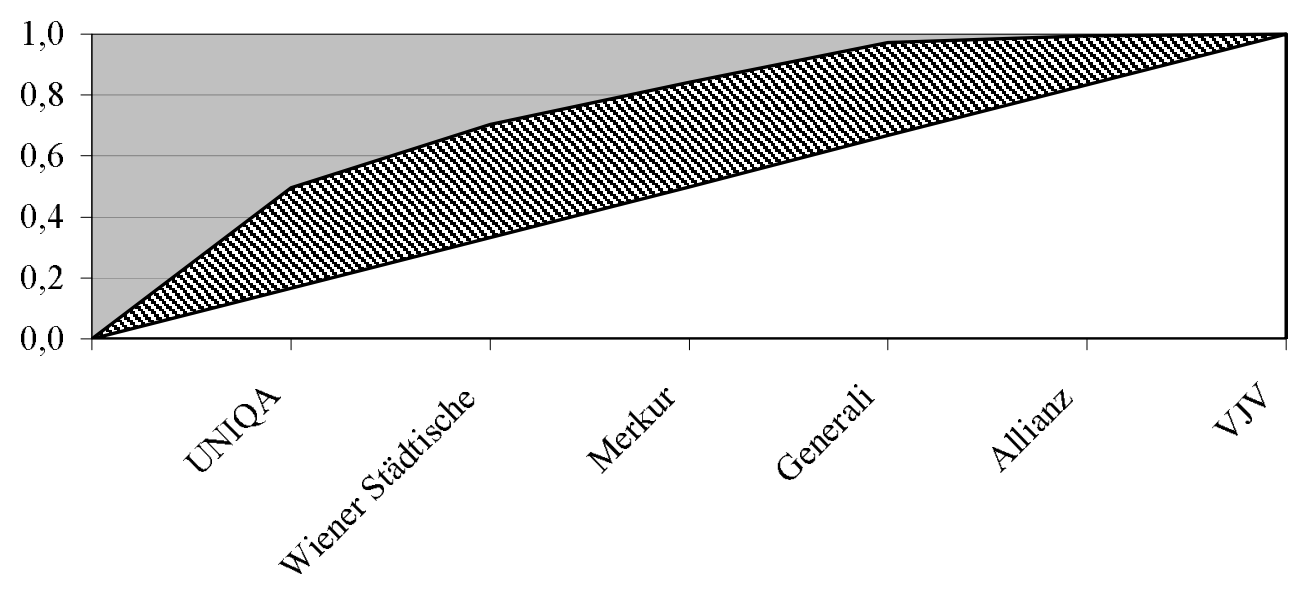

Abbildung 1: Konzentrationskurve 1992 und 2001

wenig niederschlägt. Daher erscheinen die Maßzahlen (mit Ausnahme der Konzentrationsraten) nur bedingt zur statischen Konzentrationsmessung geeignet, weil sie offensichtlich die Konzentration am Markt oft unterschätzen. Die relativ unspektakulär wirkende Konzentrationsrate erscheint am besten geeignet.

Man könnte dem Trugschluss unterliegen, dass auch das Dominanzmaß überflüssig sei, weil ja aus einem Marktanteil von über 50\% ohnehin auf eine monopolartige Struktur geschlossen werden kann. Dies wäre aber eine unzulässige Interpretation, was sich auch an einem kurzen fiktiven Zahlenbeispiel demonstrieren lässt. Dazu betrachte man die Marktsituation im Jahr 2001. Was passiert nun mit der Marktstruktur, wenn die Unternehmen Wiener Städtische und Merkur fusionieren und die sonstige Marktverteilung auf die Anbieter gleich bleibt? Das würde dazu führen, dass zwar nach wie vor die UNIQA fast die Hälfte des Marktvolumens inne hätte und damit auch Marktführer wäre, dass aber 
Tabelle 5: Maßzahlen 1992-1996

\begin{tabular}{lccccc}
\hline Jahr & 1992 & 1993 & 1994 & 1995 & 1996 \\
\hline Anzahl Unternehmen & 9 & 9 & 9 & 9 & 9 \\
Unternehmenseinheiten & 7 & 7 & 7 & 7 & 6 \\
maximale Entrophie & 1.946 & 1.946 & 1.946 & 1.946 & 1.792 \\
\hline Konzentrationsmaße & & & & & \\
CR1 & 0.325 & 0.321 & 0.319 & 0.315 & 0.511 \\
CR3 & 0.730 & 0.722 & 0.721 & 0.719 & 0.854 \\
Herfindahl $\alpha=2.5$ & 0.227 & 0.224 & 0.223 & 0.222 & 0.362 \\
Herfindahl $\alpha=2$ & 0.218 & 0.216 & 0.216 & 0.214 & 0.338 \\
Herfindahl $\alpha=1.5$ & 0.209 & 0.207 & 0.206 & 0.206 & 0.308 \\
Entropie ln & 1.630 & 1.635 & 1.637 & 1.639 & 1.299 \\
relative Entropie & 0.838 & 0.840 & 0.841 & 0.842 & 0.725 \\
Exponential & 0.196 & 0.195 & 0.195 & 0.194 & 0.273 \\
Rosenbluth & 0.241 & 0.238 & 0.237 & 0.236 & 0.345 \\
\hline Dominanzmaß & & & & & \\
Deffaa & 3 & 3 & 3 & 3 & 1 \\
\hline
\end{tabular}

Tabelle 6: Maßzahlen 1997-2001

\begin{tabular}{lccccc}
\hline Jahr & 1997 & 1998 & 1999 & 2000 & 2001 \\
\hline Anzahl Unternehmen & 9 & 9 & 8 & 8 & 8 \\
Unternehmenseinheiten & 6 & 6 & 6 & 6 & 6 \\
maximale Entropie & 1.792 & 1.792 & 1.792 & 1.792 & 1.792 \\
\hline Konzentrationsmaße & & & & & \\
CR1 & 0.508 & 0.504 & 0.500 & 0.497 & 0.495 \\
CR3 & 0.852 & 0.851 & 0.847 & 0.846 & 0.843 \\
Herfindahl $\alpha=2.5$ & 0.360 & 0.357 & 0.353 & 0.350 & 0.348 \\
Herfindahl $\alpha=2$ & 0.336 & 0.333 & 0.330 & 0.327 & 0.325 \\
Herfindahl $\alpha=1.5$ & 0.306 & 0.305 & 0.302 & 0.299 & 0.298 \\
Entropie ln & 1.304 & 1.307 & 1.315 & 1.322 & 1.325 \\
relative Entropie & 0.728 & 0.729 & 0.734 & 0.738 & 0.740 \\
Exponential & 0.272 & 0.271 & 0.268 & 0.267 & 0.266 \\
Rosenbluth & 0.344 & 0.342 & 0.339 & 0.337 & 0.336 \\
\hline Dominanzmaß & & & & & \\
Deffaa & 1 & 1 & 1 & 1 & 1 \\
\hline
\end{tabular}

trotzdem nicht mehr von einer monopolartige Struktur gesprochen werden könnte, sondern nun wieder eine oligopolartige Struktur vorliegen würde, in diesem Fall allerdings mit zwei Unternehmen (UNIQA und das "Fusionsunternehmen" aus Wiener Städtischen und Merkur). Anders ausgedrückt verwendet das Dominanzmaß in der Analyse nicht nur die Marktanteile der Unternehmen an sich, sondern berücksichtigt auch die Verhältnisse der Marktanteile zueinander, sodass eine Monopolstellung eines Unternehmens nur dann 
vorliegt, wenn der Marktanteil dieses Unternehmens hoch ist und gleichzeitig die Marktanteile der Unternehmen aus der Verfolgergruppe gering sind. Damit lässt sich aus der Kenntnis der Marktanteile relativ einfach und schnell berechnen, ob eine monopolartige oder oligopolartige Struktur am Markt vorliegt. In den Jahren 1996 bis 2001 ist die Entwicklung wieder unspektakulär, nur geringe Veränderungen traten auf.

\section{Ursachen und Folgen der Marktkonzentration}

Die private Krankenversicherung steht wie auch die soziale Krankenversicherung unter massiven Kostendruck, der einerseits auf die demographische Struktur der Versicherten und andererseits auf den medizinischen Fortschritt zurückzuführen ist. Die Bevölkerung wird insgesamt immer älter und die medizinischen Möglichkeiten immer besser, aber auch teurer. Eine Krankenversicherung bietet intertemporalen Versicherungsschutz an, was zur Folge hat, dass junge Versicherte im Verhältnis zu ihrem Krankheitsrisiko hohe Prämien einzahlen, ältere Versicherte hingegen in Relation niedrige Prämien beitragen. Bei hohen Kostensteigerungen - verursacht durch längere Lebensdauer und/oder höhere medizinische Kosten - müssen daher auf lange Sicht die Prämien angehoben werden. Andererseits sinkt mit der Preissteigerung die Nachfrage von Kunden, insbesondere natürlich von Kunden mit niedrigem Krankheitsrisiko, was wiederum eine weitere Preissteigerung notwendig machen würde. Bei Versicherungen kommt als weiterer Aspekt die Größe der Risikogemeinschaft dazu, weil eine größere Risikogemeinschaft für den Versicherer von Vorteil ist, da sich zufällige Schwankungen innerhalb der Risikogruppe ausgleichen können. Je größer also die Risikogemeinschaft, desto knapper könnte man die Prämien kalkulieren, um den Preissteigerungen entgegenwirken zu können. Daneben drückt der Wettbewerb um Marktanteile - wie auf anderen Märkten auch - die Preise und erhöht die Marktkonzentration. Das Zusammenspiel von Angebot und Nachfrage, von Nachfrage und Preis und letztendlich auch von Wettbewerb und Preispolitik funktioniert am Versicherungsmarkt aber nur sehr eingeschränkt, weil der Markt durch den Staat stark reguliert ist. Insbesondere die Preisgestaltung unterliegt strengen gesetzlichen Kriterien, sodass hier in keiner Weise ein freier Markt vorliegt. Schon 1990 merkt Peter Szopo an, dass ein unregulierter Wettbewerb auf dem Markt der Krankenversicherung nicht funktionieren würde und daher eine staatliche Aufsicht erforderlich ist (Szopo, 1990). Die staatliche Aufsicht trägt in diesem Fall sicher auch dazu bei, dass für die Versicherten keine massive Veränderungen durch die Konzentrationsverschiebung auftreten. Auch für die Mitbewerber werden sich keine Konsequenzen ergeben, denen man eindeutig nachweisen kann, dass sie sich ursächlich aus der Konzentrationsverschiebung ergeben haben. Das marktbeherrschende Unternehmen hat einen Wettbewerbsvorteil durch die größere Risikogemeinschaft, ist aber in der Preisgestaltung nicht frei und kann daher die Vorteile falls überhaupt - nur sehr eingeschränkt nutzen.

\section{Resümee}

Die private Krankenversicherung in Österreich wurde in Hinblick auf Konzentration und Marktstruktur untersucht. 1996 fand mit dem Zusammenschluss von Bundesländer und 
Austria-Collegialität zur BARC ein wesentlicher Einschnitt in die Marktstruktur statt, der zur Folge hatte, dass die Oligopolstellung der Unternehmen Bundesländer, Wiener Städtische und Austria-Collegialität von einer monopolartigen Struktur mit der BARC (jetzt UNIQA) als vorherrschendes Unternehmen abgelöst wurde. Weiters ist darauf hinzuweisen, dass nicht die Marktanteile einzelner Unternehmen alleine als Indizien für eine allfällige monopolartige Struktur verwendet werden sollten. Ein Dominanzmaß bringt zusätzliche Hinweise auf die Marktstruktur, weil auch die Relation der Marktanteile von Untenahmen berücksichtigt wird. Eine Monopolstellung in einem vollkommen freien Markt würde freie Preisfestsetzung nach sich ziehen, bei Versicherungsunternehmen ist aber davon abzuraten, eine mögliche Monopol- oder Oligopolstellung in einer Prämienfestsetzung nachvollziehen zu wollen. Der Versicherungsmarkt unterliegt in der Prämiengestaltung genauen gesetzlichen Regelungen, die im Versicherungsaufsichtsgesetz festgehalten sind. Dort heißt es, dass Prämien auf versicherungsmathematischer Grundlage unter Verwendung von Wahrscheinlichkeitstafeln und anderen einschlägigen statistischen Daten zu berechnen sind, als Aufsichtsbehörde fungiert die Finanzmarktaufsicht (früher die Versicherungsaufsicht).

Daneben unterscheiden sich die einzelnen Produkte der Versicherungsunternehmen meist in einigen Details, sodass in Praxis nur schwer feststellbar ist, ob hier eine Preisabsprache erfolgte, oder ob einfach aufgrund der gleichen Rechnungsgrundlagen ein mehr oder weniger ähnlicher Preis sozusagen zufällig entstanden ist.

\section{Quellen aus dem Internet}

- http://www.fma.gv.at/de/fma/statisti/versiche/oesterre.htm, 7.10.2005

- http://www.generali-holding.at/Daten/AT/Project/HoldingWeb/HoldingWeb.nsf /pages/GVG_Maerkte_Oesterreich_IU, 20.1.2003

- http://www.generali-holding.at/Daten/AT/Project/HoldingWeb/HoldingWeb.nsf /pages/GVG_Histor, 20.1.2003

- http://holding.generali.at, 7.10.2005

- http://www.uniqa.at/ir/unternehmen/index.html, 20.1.2003

- http://www.uniqa.at/ir/unternehmen/index.html, 7.10.2005

- http://www.wuestenrot.at/c_unternehmen_vers_zahlen.php, 7.10.2005

\section{Literatur}

Adelman, M. (1969). Comment on the "H“" Concentration Measure as a NumbersEquivalent. The Review of Economics and Statistics, 51, 99-101.

Bruckmann, G. (1969). Einige Bemerkungen zur statistischen Messung von Konzentration. Metrika, 14, 183-213.

Bundesministerium für Finanzen. (1993). Österreichische Versicherungsstatistik für das Jahr 1992. Wien: Bundesministerium für Finanzen. 
Bundesministerium für Finanzen. (1994). Österreichische Versicherungsstatistik für das Jahr 1993. Wien: Bundesministerium für Finanzen.

Bundesministerium für Finanzen. (1995). Österreichische Versicherungsstatistik für das Jahr 1994. Wien: Bundesministerium für Finanzen.

Bundesministerium für Finanzen. (1996). Österreichische Versicherungsstatistik für das Jahr 1995. Wien: Bundesministerium für Finanzen.

Bundesministerium für Finanzen. (1997). Österreichische Versicherungsstatistik für das Jahr 1996. Wien: Bundesministerium für Finanzen.

Bundesministerium für Finanzen. (1998). Österreichische Versicherungsstatistik für das Jahr 1997. Wien: Bundesministerium für Finanzen.

Bundesministerium für Finanzen. (2001). Versicherungsaufsicht: Jahresbericht 2000. Wien: Bundesministerium für Finanzen.

Deffaa, W. (1982). Die Berücksichtigung monopolistischer und oligopolistischer Strukturen in der statistischen Konzentrationsmessung. Allgemeines Statistisches Archiv, 66, 323-340.

Eurich, A., et al. (1997). Die Entwicklung der Anbieterkonzentration auf dem deutschen Erstversicherungsmarkt von 1991 bis 1994. Zeitschrift für Betriebswirtschaftslehre, 67(2), 1093-1110.

Hall, M., and Tidemann, N. (1967). Measures of concentration. Journal of the American Statistical Association, 62, 162-268.

Häni, P. K. (1987). Die Messung der Unternehmenskonzentration. Grüsch: Rüegger.

Hannah, L., and Kay, J. (1977). Concentration in Modern Industry: Theory, measurement and the U.K. experience. London, Basingstoke: Macmillan.

Hart, P. (1971). Entropy and Other Measures of Concentration. Journal of the Royal Statistical Society, Series A, 134, 73-85.

Herberg, H. (1989). Preistheorie. Eine Einführung. (2 ed.). Stuttgart, Berlin, Köln: Kohlhammer.

Horvath, J. (1970). Suggestion for a Comprehensive Measure of Concentration. Southern Economic Journal, 36, 446-452.

Kartellgesetz. (1988). (BGB1. Nr. 600/1988, zuletzt geändert durch BGB1. Nr. 033/2002)

Marfels, C. (1971). Einige neuere Entwicklungen in der Messung der industriellen Konzentration. Metrika, 17, 69-81.

Marfels, C. (1977). Erfassung und Darstellung industrieller Konzentration: statistische Grundlagen u. Mölichkeiten. Baden-Baden: Nomos-Verlag-Ges.

Piesch, W. (1975). Statistische Konzentrationsmaße: formale Eigenschaften und verteilungstheoretische Zusammenhänge. Tübingen: Mohr.

Pisch, W., and Schmidt, I. (1983). Die Verwendbarkeit von Konzentrationsmaßen in der Europäischen Wettbewerbspolitik. Luxemburg.

Rosenbluth, G. (1961). Diskussionsbeitrag in J. Niehans: Round-Table-Gespräch: Messung der industriellen Konzentration. In F. Neumark (Ed.), Die Konzentration in der Wirtschaft (p. 367-395). Berlin: Verlag von Duncker und Humbolt.

Spätling, D. (1970). Zur Messung der wirtschaftlichen Konzentration und des Wettbewerbsgrades: Über einige neuere Maße der wirtschaftlichen Konzentration. Konjunkturpolitik. Zeitschrift für angewandte Konjunkturforschung, 233-261.

Statistik Austria. (2003). Statistisches Jahrbuch 2004. Wien: Statistik Austria. 
Szopo, P. (1990). Tarifregulierung in der privaten Krankenversicherung. WIFO-Monatsberichte, 4, 260-262.

Theil, H. (1967). Economics and Information Theory. Amsterdam: North-Holland Publ. Url, T. (1995). Divergierende Entwicklung in den einzelnen Sparten der Versicherungswirtschaft. Die österreichische Privatversicherungswirtschaft 1993/94. WIFO-Monatsberichte, 7, 465-469.

Url, T. (1996). Kennzahlen zur österreichischen Versicherungswirtschaft. Wien: WIFO Österr. Inst. für Wirtschaftsforschung.

Versicherungsaufsichtsgesetz. (1978). (BGB1. Nr. 569/1978, zuletzt geändert durch BGB1. Nr. 008/2005)

Author's address:

Christine Duller

Institut für angewandte Statistik

Johannes Kepler Universität Linz

Altenbergerstrasse 69

4040 Linz

Tel. 0732 / 2468 - 9128

E-mail: christine.duller@jku.at 